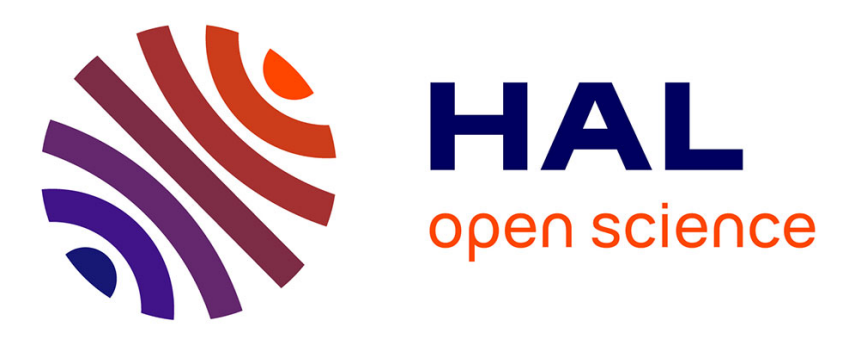

\title{
Symmetry and Golden Ratio in the Analysis of Regular Pentagon
}

\author{
Amelia Carolina Sparavigna, Mauro Maria Baldi
}

\section{To cite this version:}

Amelia Carolina Sparavigna, Mauro Maria Baldi. Symmetry and Golden Ratio in the Analysis of Regular Pentagon. 2016. hal-01283001

\section{HAL Id: hal-01283001 \\ https://hal.science/hal-01283001}

Preprint submitted on 4 Mar 2016

HAL is a multi-disciplinary open access archive for the deposit and dissemination of scientific research documents, whether they are published or not. The documents may come from teaching and research institutions in France or abroad, or from public or private research centers.
L'archive ouverte pluridisciplinaire HAL, est destinée au dépôt et à la diffusion de documents scientifiques de niveau recherche, publiés ou non, émanant des établissements d'enseignement et de recherche français ou étrangers, des laboratoires publics ou privés. 


\title{
Symmetry and Golden Ratio in the Analysis of Regular Pentagon
}

\author{
Amelia Carolina Sparavigna ${ }^{1}$ and Mauro Maria Baldi ${ }^{2}$ \\ 1 - Department of Applied Science and Technology, Politecnico di Torino, Torino, Italy \\ 2 - Department of Control and Computer Engineering, Politecnico di Torino, Torino, Italy
}

\begin{abstract}
Regular pentagon is a geometric figure which had a relevant symbolic meaning in Pythagorean and Platonic philosophies and a subsequent important role in the Western thought, appearing also in arts and architecture. A property of the pentagon, which was probably found by Pythagoreans, is that the ratio between diagonal and side is the golden ratio. Here, we will study some links between regular pentagon and golden ratio. We will focus first on the group of five-fold rotation symmetry, to find the position of the vertices of this geometric figure in the complex plane; then, we will give an analytic method to solve the same problem in the Cartesian plane. In the analytic approach, we find the golden ratio without any specific geometric consideration. This study can be interesting also for a general education in mathematics, because it can convey and link several concepts, requiring only a general pre-college education.
\end{abstract}

Keywords: Geometry, Symmetry, Mathematics, Golden Ratio, Mathematics Education

Introduction. In geometry, pentagons are five-sided polygons, which can be regular when the interior angles are equal to $\pi-2 \pi / 5$, that is, $108^{\circ}$. With regular pentagons we can construct one of the Platonic solids, the dodecahedron. Plato wrote about the solids having congruent regular polygons as faces in his Timaeus, c.360 B.C., associating them to the four classical elements (earth, air, water, and fire) [1]. The dodecahedron is the fifth solid, of which Plato told simply: "There was yet a fifth combination which God used in the delineation of the universe" [2]. Besides this role in Plato's cosmology, the regular pentagon had a general importance in geometry, art and philosophy, probably because of the fact that its diagonals are in the golden ratio to its sides. We find the pentagon, for instance, in Agrippa's De Philosophia Occulta, to illustrate the proportion, measure and harmony of human body [3].

The property that the ratio between diagonal and side is the golden ratio $\phi$, that is $\phi=(1+\sqrt{5}) / 2$, was first reported in a written text at Euclid's time. Euclid gives two constructions of regular pentagon in Book IV, as Propositions 11 \& 12. According to Sir Thomas Little Heath, who translated the Euclid's works, these methods had been probably previously developed by the Pythagoreans [4]. Besides this link to the golden ratio, another important feature related to the Pythagorean philosophy is observed in a regular pentagon: diagonals are creating another geometrical figure, the pentagram, which is the symbol of Pythagoreanism [5]. As told in [6], a striking visual feature of the regular pentagon was that its diagonals create the pentagram and, in it, a new pentagon inside the original pentagon. This self-reproductive property of the pentagon was probably evocative to Pythagoreans of the transmigration of souls [6].

With its five-fold symmetry, the pentagon attracted an artist, Albrecht Dürer, and the astronomer Johannes Kepler, for the problem of polygonal tiling of the plane using pentagons [7]. In his Harmonices Mundi, when discussing on the congruence of harmonic figures, Kepler investigated the symmetry of polyhedra and explored orderly arrangements of plane pentagons. According to [7], this was an anticipation of Penrose aperiodic tiling models, that served for the geometry of quasicrystal structures. 
Since regular pentagons and pentagrams have a large impact on arts and philosophy, we should not be surprised if the pentagon is also a symbol of the Sacred Geometry, a discipline ascribing sacred meanings to geometric shapes and proportions $[8,9]$. In a previous paper, we have already discussed one of these symbols, the Vesica Piscis, a shape coming from the intersection of two circles having the same radius, arranged so that the center of each circle lies on the perimeter of the other [10]. Here, we will discuss some properties of the regular pentagon; as we did in [10], we will focus our analysis on the algebra that we can obtain from the symbol. We will see that the study of such symbol can be interesting also for a general education in mathematics, because it can convey several concepts of mathematics to people having just a pre-college education. In particular, we will link the symmetry of the pentagon to an analytic method to solve it, demonstrating some properties linking the pentagon to the golden ratio. Let us start our discussion from the symmetry of pentagon (with its five-fold rotation group), a symmetry which is also observed in nature (Fig.1).

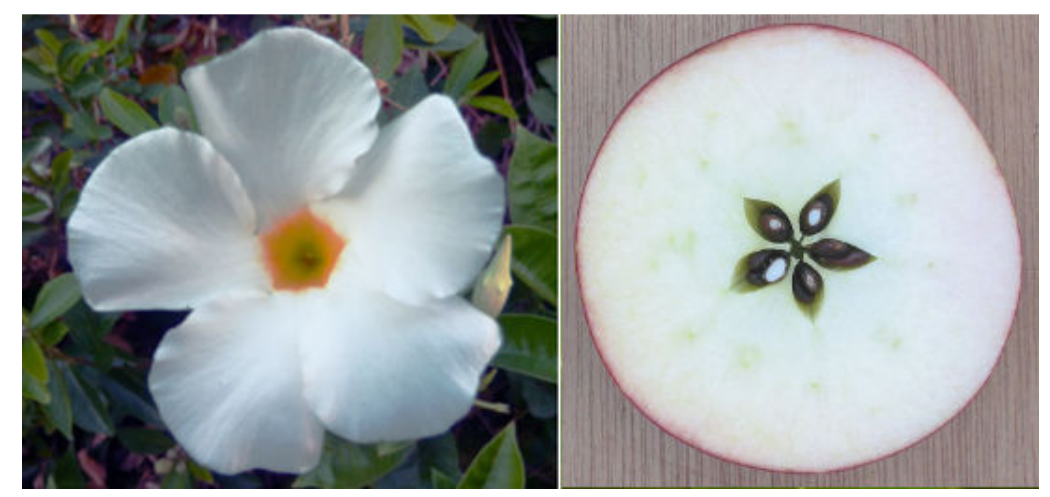

Figure 1: The five-fold symmetry (pentamerism) is common in nature. Many flowers possess it as shown by the picture on the left. Note the pentagon in the central part of the flower. On the right, pentamerism in an apple (Courtesy: Rasbak, Wikipedia).

The five-fold symmetry. The regular pentagon possesses a five-fold symmetry, that is, a rotational symmetry of order 5 , given by rotations in the plane of the polygon about its center of angles $2 \pi / 5$, $4 \pi / 5,6 \pi / 5$ and $8 \pi / 5$. The rotation of $10 \pi / 5=2 \pi$ is the identity. It has also five lines of reflection symmetry.

A symmetry is coming from a transformation of a planar figure, which is a rigid motion that leaves the figure unchanged. New transformations can be obtained by following one symmetry after another. In this way, an algebra of symmetries is obtained, where the multiplication is given by the composition of symmetries. This algebra contains the identity element and the inverse elements. In other words, we have a symmetry group for each geometric figure, because every figure has at least the symmetry group consisting only of the identity. Let us consider an example; a rectangle, which is not a square, has four elements in its symmetry group, namely rotations about an axis perpendicular to the figure and passing through its center, of angles 0 and $\pi$, and a horizontal and vertical flip about the midlines. This is the Klein 4-group. A square, which is more symmetric, has 8 elements in its symmetry group.

Let us remember that a regular polygon has all its sides having the same length and all its angles the same value. Then, we can have an equilateral triangle, which is a regular 3-gon, a square which is a regular 4-gon, a pentagon a regular 5-gon and so on. The symmetry group of a regular $n$-gon has $n$ rotational and $n$ reflection symmetries, for a total of $2 n$ symmetries. It is called the dihedral group of the $n$-gon and denoted by $D_{n}$. The pentagon has a dihedral group of 10 elements. There are also two subgroups, consisting of the five rotation symmetries and the subgroup of the five mirror symmetries. 
Here, we consider a representation of the rotation group of the pentagon in a complex plane. This plane is a geometric representation of the complex numbers established by the real axis and the orthogonal imaginary axis. The position of the vertices of a pentagon can be described by complex numbers, which can be assumed in a rectangular form, $(a+i b)$, or in a polar form or in the exponential form. This last form is giving the position of a point $Z$ in the complex plane by the Euler's identity: $Z=A e^{i \theta}=A(\cos \theta+i \sin \theta)$.

Using the pentagon in Figure 2(a), inscribed in a circle of unit radius, positions of vertices $\mathrm{O}, \mathrm{A}, \mathrm{B}$, $\mathrm{C}$ and $\mathrm{D}$ are given by the following numbers: $1, e^{i 2 \pi / 5}, e^{i 4 \pi / 5}, e^{i 6 \pi / 5}, e^{i 8 \pi / 5}$. These numbers are also the representation of the elements of rotation group, that, with the multiplication, give us an algebra. Here two examples of this algebra: let us consider two elements of the group, for instance $e^{i 2 \pi / 5}, e^{i 4 \pi / 5}$ and multiply them. We have: $e^{i 2 \pi / 5} \cdot e^{i 4 \pi / 5}=e^{i 2 \pi / 5+i 4 \pi / 5}=e^{i 6 \pi / 5}$, which is another element of the group. If we consider $e^{i 6 \pi / 5}, e^{i 8 \pi / 5}$ we have $e^{i 6 \pi / 5} \cdot e^{i 8 \pi / 5}=e^{i 14 \pi / 5}=e^{i 10 \pi / 5} \cdot e^{i 4 \pi / 5}=e^{i 4 \pi / 5}$, because of the identity $1=e^{i 10 \pi / 5}$. Inverse elements of the group are $e^{-i 2 \pi / 5}, e^{-i 4 \pi / 5}, e^{-i 6 \pi / 5}, e^{-i 8 \pi / 5}$.
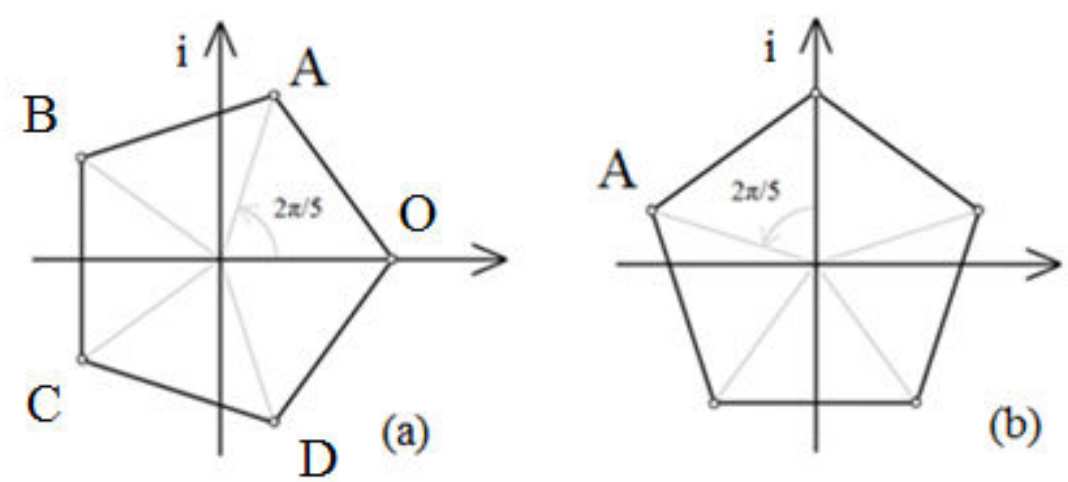

Figure 2: Positions of the vertices in the complex plane for a pentagon (a), and the same figure rotated of 90 degrees (b).

Let us consider diagonal AD and side BC. Using Euler's identity, the ratio between their lengths in the complex plane has the value:

$$
\phi=\frac{\overline{A D}}{\overline{B C}}=\frac{2 \sin \frac{2 \pi}{5}}{2 \sin \frac{4 \pi}{5}}=\frac{\sin \frac{2 \pi}{5}}{\sin \left(\pi-\frac{\pi}{5}\right)}=\frac{2 \sin \frac{\pi}{5} \cos \frac{\pi}{5}}{\sin \frac{\pi}{5}}=2 \cos \frac{\pi}{5}
$$

It means that, following Euclid who told that the ratio between diagonal and side is the golden ratio, we have that $\phi$ is two times the cosine of $\pi / 5$. However, from Figure 2(a), we have also immediately that the apothem of the regular pentagon, inscribed in the goniometric circumference, is equal to the cosine of $\pi / 5$ and then it is one-half the golden ratio.

Let us conclude considering the rectangular representation of the point $\mathrm{A}$ with $e^{i 2 \pi / 5}$. It is given by: 


$$
\begin{aligned}
e^{i 2 \pi / 5} & =\cos \frac{2 \pi}{5}+i \sin \frac{2 \pi}{5}=\frac{-1+\sqrt{5}}{4}+i \frac{\sqrt{10+2 \sqrt{5}}}{4}=\frac{\sqrt{(-1+\sqrt{5})^{2}}}{4}+i \frac{\sqrt{10+2 \sqrt{5}}}{4} \\
& =\frac{\sqrt{6-2 \sqrt{5}}}{4}+i \frac{\sqrt{10+2 \sqrt{5}}}{4}=\frac{1}{2} \sqrt{\frac{3-\sqrt{5}}{2}}+i \frac{1}{2} \sqrt{\frac{5+\sqrt{5}}{2}}
\end{aligned}
$$

We can repeat the same calculation for the other four vertices.

However, pentagon can appear as in the Figure 2(b), rotated by 90 degrees. We have that the position of $\mathrm{A}$ is now given by:

$$
e^{i \pi / 2} \cdot e^{i 2 \pi / 5}=i \cos \frac{2 \pi}{5}-\sin \frac{2 \pi}{5}=-\frac{1}{2} \sqrt{\frac{5+\sqrt{5}}{2}}+i \frac{1}{2} \sqrt{\frac{3-\sqrt{5}}{2}}
$$

And so on, for all the vertices.

Golden ratio. To have equation (2), we need to use a property of the remarkable angle $\gamma=\pi / 5=$ $2 \pi / 10=36^{\circ}$. Let us define $\eta=2 \gamma=72^{\circ}$, and consider Figure 3 .

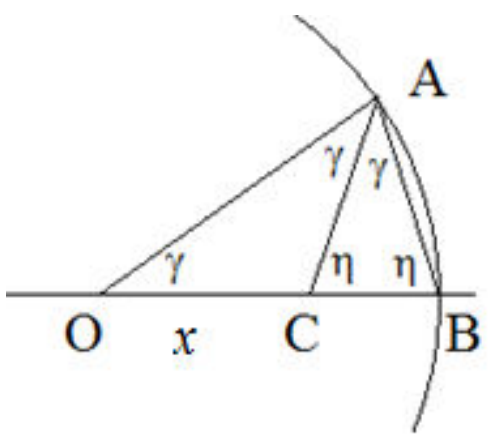

Figure 3: $A B=O C=x$ is the golden ratio of radius $O B$.

Triangle $O A B$ is isosceles, being $O A$ and $O B$ two radii of a goniometric circumference. $A C$ is bisecting angle $\mathrm{OA} B$. From triangle $A B C$; we have that $B \hat{A} C=\gamma=2 \pi / 10, A \hat{B} O=2 \gamma=\eta=4 \pi / 10$, then $A \hat{C} B=\pi-\gamma-\eta=\pi-2 \pi / 10-4 \pi / 10=\eta$. So, we have that triangle $A B C$ is isosceles. Triangles $O A B$ and $A B C$ have congruent angles, therefore they are similar. We have: $O A / A B=A B / B C$. Since $A B=A C=O C=x$ and $O A=O B=1$, we have:

$$
\phi=\frac{1}{x}=\frac{x}{(1-x)}
$$

Eq.4 is the equation that defines the golden ratio $\phi$. This means that $A B=O C$ is the golden ratio of the radius. From (4), we have $x^{2}=(1-x)$ and $x=(-1+\sqrt{5}) / 2$. Then: $\phi=1 / x=(1+\sqrt{5}) / 2$. However, we need sine and cosine of $4 \pi / 10$. We have that 
$\sin (4 \pi / 10)=\cos (\pi / 2-4 \pi / 10)=\cos (\pi / 10)$ and $\cos (4 \pi / 10)=\sin (\pi / 10)$. From the Figure 3, $\sin (\pi / 10)=A B / 2=x / 2$, then it is equal to $(-1+\sqrt{5}) / 4$. From this result, (2) had been evaluated.

Using the rotation group and complex numbers, and also a little bit of geometry concerning the golden ratio, we have seen how to find the vertices of regular pentagon. From them, all the other properties can be obtained.

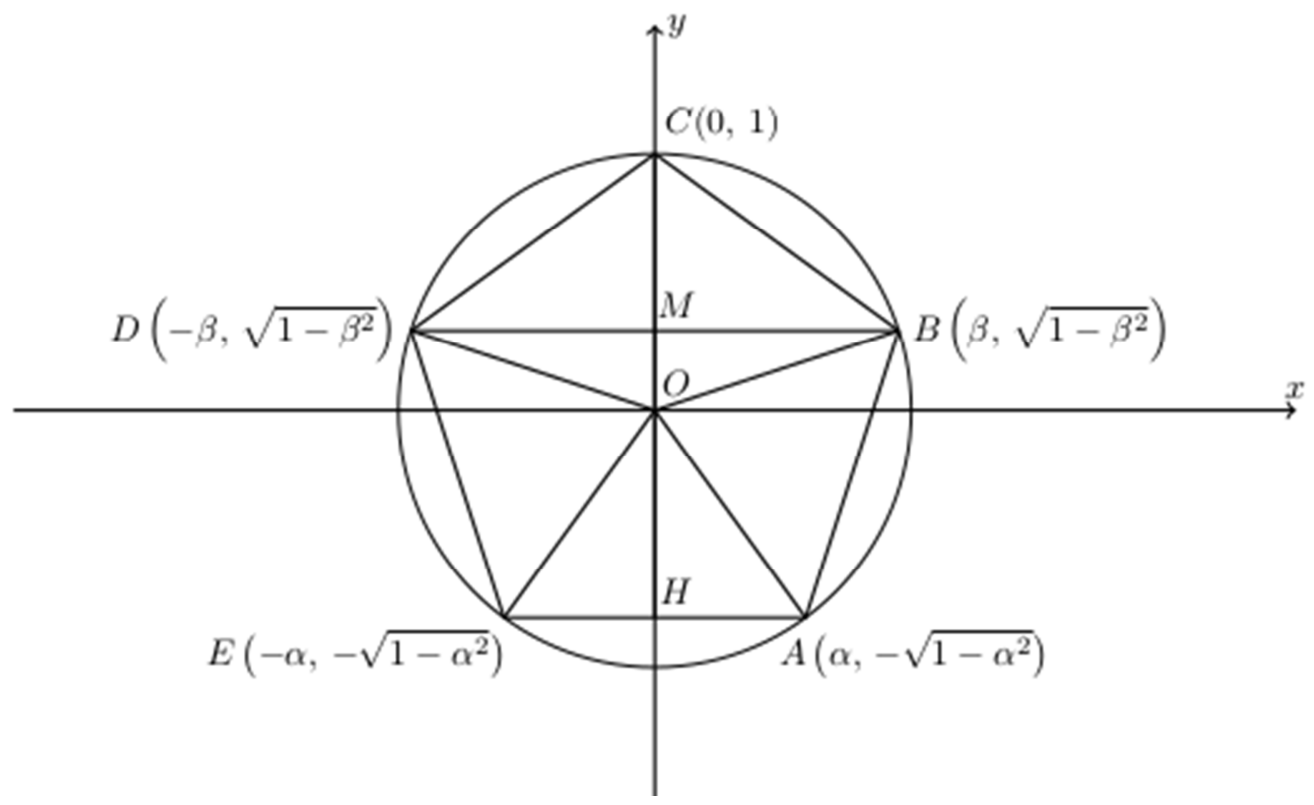

Figure 4: Coordinates of vertices in the Cartesian plane, for a pentagon inscribed in a circumference of radius 1 . Note that $\overline{A E}=2 \alpha, \overline{B D}=2 \beta$.

The analytic method. Here, our aim is linking the algebra from symmetry to an analytic method for finding the positions of vertices. Let us refer to the Figure 4 for calculation. From this Figure, we have that $0<\alpha<\beta<1$. Since the pentagon is regular, it must be $\overline{A B}=\overline{B C}$. In terms of coordinates of points $A, B$ and $C, \overline{A B}=\overline{B C}$ becomes:

$$
\left((\alpha-\beta)^{2}+\left(\sqrt{1-\alpha^{2}}+\sqrt{1-\beta^{2}}\right)^{2}\right)^{1 / 2}=\left(\beta^{2}+\left(1-\sqrt{1-\beta^{2}}\right)^{2}\right)^{1 / 2}
$$

If we square both sides of this equation, we have to guarantee that one of the two radicands must be non-negative, in order to avoid the introduction infeasibility due to the squaring operations. In other words, we have to solve the following system:

$$
\left\{\begin{array}{l}
(\alpha-\beta)^{2}+\left(\sqrt{1-\alpha^{2}}+\sqrt{1-\beta^{2}}\right)^{2}=\beta^{2}+\left(1-\sqrt{1-\beta^{2}}\right)^{2} \\
(\alpha-\beta)^{2}+\left(\sqrt{1-\alpha^{2}}+\sqrt{1-\beta^{2}}\right)^{2} \geq 0
\end{array}\right.
$$


(b) is always satisfied, because it is the sum of squared quantities. Thus we have to consider (a), that is:

$$
(\alpha-\beta)^{2}+\left(\sqrt{1-\alpha^{2}}+\sqrt{1-\beta^{2}}\right)^{2}=\beta^{2}+\left(1-\sqrt{1-\beta^{2}}\right)^{2}
$$

Let us also consider that the regular pentagon is made of five congruent triangles. Triangles are congruent when they have exactly the same sides and angles. In this manner, a triangle can become another triangle using turns and flips. In the case of Figure 4, we can start from triangle $A O E$ and, after a rotation, having triangle $A O B$. Then, in the same manner, we can have $B O C$. In fact, as we have discussed before, the regular pentagon has an order of rotation symmetry equal to 5 . Since $A O E$ is isomeric to $B O C$, the areas of the two triangles are equal. Thus:

$$
\frac{1}{2} \overline{A E} \cdot \overline{H O}=\frac{1}{2} \overline{O C} \cdot \overline{M B} \Rightarrow \frac{1}{2} \cdot(2 \alpha) \cdot \sqrt{1-\alpha^{2}}=\frac{1}{2} \cdot 1 \cdot \beta \Rightarrow \beta=2 \alpha \cdot \sqrt{1-\alpha^{2}}
$$

We have two equations (5) and (6) and two unknown variables $\alpha, \beta$.

$$
\left\{\begin{array}{l}
(\alpha-\beta)^{2}+\left(\sqrt{1-\alpha^{2}}+\sqrt{1-\beta^{2}}\right)^{2}=\beta^{2}+\left(1-\sqrt{1-\beta^{2}}\right)^{2} \\
\beta=2 \alpha \cdot \sqrt{1-\alpha^{2}}
\end{array}\right.
$$

After simple passages:

$$
\left\{\begin{array}{l}
\sqrt{\left(1-\alpha^{2}\right)\left(1-\beta^{2}\right)}=\alpha \beta-\sqrt{1-\beta^{2}} \\
\beta=2 \alpha \cdot \sqrt{1-\alpha^{2}}
\end{array}\right.
$$

A shortcut in the resolution of this equation is the squaring of both sides. The squaring operation gives no problems; however, it must be performed with care. Since $\alpha>0$, we have that:

$$
\sqrt{1-\alpha^{2}}=\frac{\beta}{2 \alpha}
$$

If we square both sides of this equation, we also have to guarantee that $\beta / 2 \alpha \geq 0$; this condition is already satisfied because, by construction, we have that $\alpha, \beta>0$.

Therefore, from the second equation in (8), we have: 


$$
\beta^{2}=4 \alpha^{2}\left(1-\alpha^{2}\right)=4 \alpha^{2}-4 \alpha^{4} \Rightarrow 1-\beta^{2}=1-4 \alpha^{2}+4 \alpha^{4}=\left(1-2 \alpha^{2}\right)^{2}
$$

Using (9) in the first equation of (8), with the substitution $\beta=2 \alpha \cdot \sqrt{1-\alpha^{2}}$, we have a noteworthy simplification. However, radicals in (8) must be handled with caution. In fact we have that:

$$
\left\{\begin{array}{l}
\left|1-2 \alpha^{2}\right| \sqrt{1-\alpha^{2}}=2 \alpha^{2} \cdot \sqrt{1-\alpha^{2}}-\left|1-2 \alpha^{2}\right| \\
\beta=2 \alpha \cdot \sqrt{1-\alpha^{2}}
\end{array}\right.
$$

It is therefore necessary to distinguish two possible cases: $1-2 \alpha^{2}<0$ or $1-2 \alpha^{2}>0$. Let us consider the second case which is that fitting our assumptions (the first case is discussed in the Appendix). (10) becomes:

$$
\left\{\begin{array}{l}
\left(1-2 \alpha^{2}\right) \sqrt{1-\alpha^{2}}=2 \alpha^{2} \cdot \sqrt{1-\alpha^{2}}-\left(1-2 \alpha^{2}\right) \\
\beta=2 \alpha \cdot \sqrt{1-\alpha^{2}}
\end{array}\right.
$$

In the first equation of (11), we can use the substitution $t=\alpha^{2}$. The system becomes:

$$
:\left\{\begin{array}{l}
(4 t-1) \sqrt{1-t}=1-2 t \\
\beta=2 \sqrt{t(1-t)} \\
1-2 t \geq 0
\end{array}\right.
$$

Squaring (*) requires the following condition: $(4 t-1 \geq 0 \wedge 1-2 t \geq 0) \vee(4 t-1<0 \wedge 1-2 t<0)$. The second part of the condition cannot be satisfied because it violates assumption $(* *)$. Therefore, squaring both sides of $(*)$ implies the resolution of the following system:

$$
\left\{\begin{array}{l}
(4 t-1)^{2}(1-t)=(1-2 t)^{2} \\
\beta=2 \sqrt{t(1-t)} \\
\frac{1}{4} \leq t \leq \frac{1}{2}
\end{array}\right.
$$

After some passages we have:

$$
t\left(16 t^{2}-20 t+5\right)=0
$$


Equation (13) has three solutions. One solution $(t=0)$ implies $\alpha=0$, which is in contrast with assumption $0<\alpha<1$. The other two solutions are:

$$
t=\frac{5 \pm \sqrt{5}}{8}
$$

Root $t=\frac{5+\sqrt{5}}{8}$ cannot be accepted because violating condition $\frac{1}{4} \leq t \leq \frac{1}{2}$, which is satisfied by $t=\frac{5-\sqrt{5}}{8}$.

After some simple passages, we have the desired coordinates of the five vertices of pentagon:

$$
\begin{aligned}
A\left(\alpha,-\sqrt{1-\alpha^{2}}\right) & =\left(\frac{1}{2} \sqrt{\frac{5-\sqrt{5}}{2}},-\frac{1}{2} \sqrt{\frac{3+\sqrt{5}}{2}}\right) \\
B\left(\beta, \sqrt{1-\beta^{2}}\right) & =\left(\frac{1}{2} \sqrt{\frac{5+\sqrt{5}}{2}}, \frac{1}{2} \sqrt{\frac{3-\sqrt{5}}{2}}\right) \\
C(0,1) & =(0,1) \\
D\left(-\beta, \sqrt{1-\beta^{2}}\right) & =\left(-\frac{1}{2} \sqrt{\frac{5+\sqrt{5}}{2}}, \frac{1}{2} \sqrt{\frac{3-\sqrt{5}}{2}}\right) \\
E\left(-\alpha,-\sqrt{1-\alpha^{2}}\right) & =\left(-\frac{1}{2} \sqrt{\frac{5-\sqrt{5}}{2}},-\frac{1}{2} \sqrt{\frac{3+\sqrt{5}}{2}}\right)
\end{aligned}
$$

Note the remarkable power of the analytic method; we have obtained the positions in the Cartesian reference, without needing sine and cosine of angles or the golden ratio.

The apothem and the golden ratio. In the previous discussion, the golden ratio does not explicitly appear. However, it must be obtained also from the analytic calculation.

And in fact, let us evaluate the ratio between diagonal $d$ and side $l$ of the pentagon (see Figure 4):

$$
\frac{d}{l}=\frac{\overline{B D}}{\overline{A E}}=\frac{2 \beta}{2 \alpha}=\frac{\beta}{\alpha}=\sqrt{\frac{5+\sqrt{5}}{5-\sqrt{5}}}=\sqrt{\frac{(5+\sqrt{5})^{2}}{20}}=\frac{5+\sqrt{5}}{2 \sqrt{5}}=\frac{1+\sqrt{5}}{2}=\phi
$$

(16) shows that the ratio between diagonal and side is the golden ratio.

Moreover, in the analysis of the previous section, we have found a relevant square root, which is also the length of $\mathrm{OH}$, the apothem of the regular pentagon (see Figure 4): 


$$
\overline{O H}=a=\sqrt{1-\alpha^{2}}=\frac{1}{2} \sqrt{\frac{3+\sqrt{5}}{2}}=\frac{\sqrt{2}}{4} \sqrt{3+\sqrt{5}}
$$

It is easy to verify that the following identity exists:

$$
\sqrt{A \pm \sqrt{B}}=\sqrt{\frac{A+\sqrt{A^{2}-B}}{2}} \pm \sqrt{\frac{A-\sqrt{A^{2}-B}}{2}}
$$

Squaring the members of (18), we have:

$$
A \pm \sqrt{B}=\frac{A}{2}+\frac{\sqrt{A^{2}-B}}{2}+\frac{A}{2}-\frac{\sqrt{A^{2}-B}}{2} \pm 2 \sqrt{\frac{\left(A+\sqrt{A^{2}-B}\right)\left(A-\sqrt{A^{2}-B}\right)}{4}}
$$

If we use the identity (18):

$$
\begin{aligned}
a & =\frac{\sqrt{2}}{4} \sqrt{3+\sqrt{5}}=\frac{\sqrt{2}}{4} \sqrt{\frac{3+\sqrt{9-5}}{2}}+\frac{\sqrt{2}}{4} \sqrt{\frac{3-\sqrt{9-5}}{2}}=\frac{\sqrt{2}}{4} \sqrt{\frac{3+\sqrt{4}}{2}}+\frac{\sqrt{2}}{4} \sqrt{\frac{3-\sqrt{4}}{2}} \\
& =\frac{\sqrt{2}}{4} \sqrt{\frac{5}{2}}+\frac{\sqrt{2}}{4} \sqrt{\frac{1}{2}}=\frac{\sqrt{5}}{4}+\frac{1}{4}=\frac{1}{2} \cdot \frac{1+\sqrt{5}}{2}=\frac{\phi}{2}
\end{aligned}
$$

Alternatively, recalling that the golden ratio $\phi$ is the positive root of equation $\phi^{2}-\phi-1=0$ or $\phi^{2}=\phi+1$. Therefore, we have:

$$
a=\frac{\sqrt{2}}{4} \sqrt{3+\sqrt{5}}=\frac{\sqrt{2}}{4} \sqrt{2+1+\sqrt{5}}=\frac{1}{2} \sqrt{1+\frac{1+\sqrt{5}}{2}}=\frac{1}{2} \sqrt{1+\phi}=\frac{1}{2} \sqrt{\phi^{2}}=\frac{\phi}{2}
$$

Conclusion. In this paper we have discussed some properties of the regular pentagon, focusing first on its group of rotation symmetry, to find the position of the vertices in the complex plane, then on an analytic method to solve the same problem in the Cartesian plane. In the solution of the analytic method, we do not need the use of trigonometric functions or geometry for the golden ratio $\phi$, if this ratio is assumed as solution of equation $\phi^{2}-\phi-1=0$. We have also shown, from the analytic method, that the ratio between the apothem of a regular pentagon and the radius of its circumscribed circumference is one-half the golden ratio.

Appendix. For Eq.(10), it is necessary to distinguish two possible cases: $1-2 \alpha^{2}<0$ or $1-2 \alpha^{2}>0$. The second case was considered in the paper. Here we discuss the first case; (10) becomes: 


$$
\left\{\begin{array}{l}
\left(-1+2 \alpha^{2}\right) \sqrt{1-\alpha^{2}}=2 \alpha^{2} \cdot \sqrt{1-\alpha^{2}}+\left(1-2 \alpha^{2}\right) \\
\beta=2 \alpha \cdot \sqrt{1-\alpha^{2}} \\
1-2 \alpha^{2} \leq 0
\end{array}\right.
$$

After some calculations, we find:

$$
2 \alpha^{2}-1=\sqrt{1-\alpha^{2}} \Rightarrow \alpha^{2}\left(4 \alpha^{2}-3\right)=0
$$

This equation has three solutions: $0, \pm \sqrt{3} / 2$. Zero and the negative solution cannot be acceptable, because we are requiring $\alpha>0$. If we use the positive solution, we find after some calculations that $\beta= \pm \sqrt{3} / 2$. The negative root is not acceptable. But also the positive, because we should have $\alpha=\beta=\sqrt{3} / 2$, which violates the hypothesis $0<\alpha<\beta<1$.

\section{References}

[1] Kotrč RF. The Dodecahedron in Plato's "Timaeus", Rheinisches Museum für Philologie, Neue Folge, 1981, 124. Bd., H. 3/4, 212-222, J.D. Sauerländers Verlag, URL: http://www.jstor.org/stable/41245051

[2] Plato’s Timaeus, Translated by B. Jowett, URL: http://classics.mit.edu/Plato/timaeus.html

[3] Heinrich Cornelius Agrippa von Nettesheim (1486 - 1535) was a German Polymath, physician, legal scholar, soldier, theologian and an occult writer. He used a regular pentagram for the proportion of the man's body in De Philosophia Occulta, Book II, Ch. 27.

[4] Heath TL. The thirteen books of Euclid's Elements (Cambridge: Cambridge University Press, 1908. URL: https://archive.org/details/euclid_heath_2nd_ed

[5] Ghyka MC. The geometry of art and life. Courier Corporation, 1946.

[6] Fossa JA. On the pentagram as a Pythagorean emblem. Revista Brasileira de História da Matemática, 2006, 6(12).

[7] Caspar LD, Fontano E. Five-fold symmetry in crystalline quasicrystal lattices, Proc. Natl. Acad. Sci. USA, 1996, 93, 14271-14278.

[8] Skinner S. Sacred Geometry: Deciphering the Code. Sterling Publishing Company, Inc., 2009. ISBN-13: 978-1402765827.

[9] Lawlor R (1982). Sacred Geometry: Philosophy and Practice (Vol. 4). New York: Thames and Hudson, 1982. ISBN-10: 0500810303, ISBN-13: 978-0500810309

[10] Sparavigna AC, Baldi MM. A Mathematical Study of a Symbol: the Vesica Piscis of Sacred Geometry. 2016 PHILICA.COM Article number 560. 